\title{
Psychophysical influences on the validity of anomaloscopic assessments of color vision
}

\author{
JeNNIFER A. STILlMAN \\ Massey University Albany, Auckland, New Zealand
}

\begin{abstract}
Anomaloscopes are used in clinical and research applications involving the assessment of color vision. Output data include the matching range (MR), the midpoint, and the anomaly quotient (AQ). The latter is commonly used to compare data obtained using different instruments. However, the midpoint and AQ ultimately depend on the MR, for which there is no universal operational definition. In this study, 5 volunteers with normal color vision each completed 510 trials in a rating task with an anomaloscope employing the Moreland equation. The aim was to investigate the nature of the perceptual transition into, and out of, a metameric match. This was found to be less than abrupt, providing ample opportunity for the influence of individual response biases. Consequently, an agreed-upon definition of the MR, associated with an appropriate psychophysical technique, would facilitate the identification and tracking over time of acquired color vision deficits and would improve the validity of interstudy comparisons.
\end{abstract}

A number of anomaloscopes are commercially available for clinical and research applications in which the assessment of color vision is required. Some instruments utilize only the red-green Rayleigh equation, whereas others also employ the blue-green Moreland equation. These instruments facilitate the diagnosis of congenital color vision deficits; however, the extent to which they allow valid comparisons among studies in which different instruments are used, or provide sufficient accuracy to track subtle changes in color vision occurring over time, is less certain. Nousiainen, Kälviäinen, and Mäntyjärvi (2000) reported that, in their investigation of color vision deficiencies associated with treatment for epilepsy, an anomaloscope (Color Vision Meter 712) did not seem efficient in detecting patients with acquired color vision abnormalities. Even if it is assumed that different instruments employ comparable optics and instantiate the appropriate equations equivalently, the reported outcomes may be affected by the variety of psychophysical procedures and operational definitions used to determine the parameters of interest. The output data generally include the matching range (MR), the midpoint, and the anomaly quotient (AQ). The AQ divides the ratio of the fixed and variable wavelengths for the current observer by that obtained for a normative group at the midpoint of the MR (National Academy of Sciences [NAS], 1981). This measure is commonly used to compare data obtained from studies in which different instruments have been used. However, the midpoint and, therefore, the AQ ultimately depend on the MR, which, for the reasons mentioned above, may vary not only between instruments, but also between sessions with the same observer.
Concerns of this nature arose during an investigation of color vision and contrast sensitivity in dental professionals, where it was considered necessary to examine the validity of the range of blue-green metameric matches reported by an anomaloscope utilizing the Moreland equation (Stillman, 2007). From the observer's point of view, the display, created with different light mixtures on either side of a circular field, is experienced either as a single-hued circle or as possessing different hues on either side of center. With the Roland anomaloscope, in an automatic diagnostic mode, stimuli on each trial are chosen by the software and are presented to the observers for simple same or different judgments, and in other modes (manual or dynamic), manual adjustments are made by the researcher or the observer to establish a match. The method of adjustment is commonly used by other instruments as well-for example, the Nagel anomaloscope (see Squire, Rodriguez-Carmona, Evans, \& Barbur, 2005) and the Oculus Heidelberg-Multi-Color (HMC) anomaloscope. The procedure and scoring with the Color Vision Meter 712 anomaloscope (Interzeag, Schlieren, Switzerland) and the Pickford-Nicholson anomaloscope (NAS, 1981) are more sophisticated. With the Interzeag instrument, data are analyzed using two psychometric functions, as recommended by Pelizzone, Sommerhalder, Roth, and Hermès (1993).

Whether observers are required to report same or different, or are required to adjust the stimulus to provide a subjective match, response bias is likely to influence the results, particularly with small numbers of trials for stimuli close to the matching region. For instance, the amount of attention paid to minor differences and the degree of

J. A. Stillman, j.a.stillman@massey.ac.nz 
concordance considered deserving of the label match will vary among individuals. Perceptually equivalent events may be reported as a match by one observer, but not by another. Such problems are, of course, well known to psychophysicists. Comparisons among individuals, or between studies, may be questionable if the psychophysical procedures employed differ or are inadequate. The potential for response bias or experimental error will depend, in part, on the nature of the transition from matching to nonmatching, with more variable responses being expected if the transition is gradual, involving a significant region of uncertainty. In the present study, the responses of 5 individuals to stimuli in the blue-green range were examined, using a Roland IF-II anomaloscope in conjunction with a rating task. The intention was to explore the nature of the transition from matching to nonmatching and vice versa. To anticipate the outcome, the study revealed a gradual transition, suggesting the need for a universally agreed upon operational definition of the MR, upon which the midpoint and the AQ depend.

\section{METHOD}

\section{Subjects}

Five volunteers, 1 man and 4 women, between 21 and 41 years of age, took part in the study; each had normal color vision as assessed using the Farnsworth-Munsell 100-Hue test.

\section{Apparatus and Procedure}

Monocular judgments were performed using the Roland IF-II anomaloscope. Initial assessments (average of three) were obtained using the automatic mode of the device for the assessment of acquired color vision deficits. The stimuli for judgment were presented as a $2^{\circ}$ circular bipartite field with a constant stimulus on the righthand side (desaturated blue, 480 and $580 \mathrm{~nm}$ ) and a variable stimulus on the left-hand side (green, $490 \mathrm{~nm}$; blue, $436 \mathrm{~nm}$ ). In the automatic mode, the amount of green in the comparison is varied between $1 \%$ and $99 \%$, and alternate trials converge on $50 \%$ green from opposite ends of the presentation range. The observer's task was to register whether the two halves of the bipartite field were of the same or different hues, and the device usually outputted a result after between 30 and 40 trials. The calculated midpoint of the matching range was expected to be at around $50 \%$ green.

Following the automatic determinations, the anomaloscope was used in its manual mode, with 30 trials at each of 17 green/blue comparison stimulus values presented for judgment in random order over a number of sessions, making a total of 510 trials. The percentage of green in the mixture was varied in fifteen 5\% steps from $15 \%$ to $85 \%$ green. In addition, to ensure an adequate number of trials in the matching range, 30 trials each of $48 \%$ and $52 \%$ green were included. The brightness and saturation values arranged by the device in the automatic mode were adopted at each step. The observers adjusted the focus of the eyepiece manually prior to testing. They were instructed not to view the field for more than a few seconds before deciding upon a rating and, if necessary, to turn away before viewing the field afresh. Settings for each trial were made by the researcher while the observer looked away. Because the machine beeped when adjusted, otherwise unnecessary adjustment sequences were used, to avoid providing clues to the degree of adjustment undertaken.

The observers rated their certainty that the two halves of the bipartite field were of the same or different hues by using the following scale: 1, certain different; 2 , fairly certain different; 3 , unsure, probably different; 4 , unsure, probably same; 5 , fairly certain same;
6 , certain same. It was expected that the rating responses would reflect changes in the perceived hue of various wavelength mixtures and could then be examined for concordance with the width, location, and symmetry of the MR, and in order to extract information about individual response biases.

\section{RESULTS AND DISCUSSION}

The relationship between the average category ratings from individual observers and the proportion of green in the variable stimulus is displayed in the panels of Figure 1. The dotted vertical lines mark the edges of the MR reported by the anomaloscope in its automatic mode. The dashed horizontal lines delineate the equivocal rating categories unsure, probably different (rating 3) and unsure, probably same (rating 4).

The systematic changes in the average category ratings from each observer suggest a gradual, rather than an abrupt, change in the similarity between the two halves of the bipartite observation field as the wavelength composition of the comparison is varied. It is of interest that the rating responses are fairly symmetrical around a maximum that, apart from a minor exception for $\mathrm{S} 3$, occurs at $50 \%$ green, the expected midpoint of the MR for normal observers. For each observer, the variability of ratings, represented by +1 standard deviation from each mean, is fairly similar at equivalent distances from the peak of the rating curves and clearly diminishes as the difference in wavelength composition increases. Certainty as to difference appears to be greater than certainty as to equivalence. The variability close to the peak of each curve implies that the stimuli in this region were rarely unequivocally identi$\mathrm{cal}$ in hue to the constant stimulus. The rating responses of S1 and S3 are remarkably similar, despite the fact that $\mathrm{S} 1$ had the narrowest assessed MR and S3 had the widest. In general, the rating responses do not correspond tightly to the width, symmetry, and location of the reported MRs, which were not always centered on the values for which the observer expressed the greatest confidence that the stimuli were the same.

Figure 1 highlights the problems inherent in quantifying subjective impressions. It cannot be assumed that differences among the widths of the rating functions reflect actual perceptual differences. Individual response biases with respect to the use of the rating scale are evident. Most of the average ratings for Subjects 1 and 2 were biased toward the response categories that included the word different. In other words, they were reluctant to use ratings that included the word same, even for stimuli in the region expected to produce a metameric match. Subjects 4 and 5 rarely used the equivocal category 4 (unsure, probably same), although their strongest ratings of sameness were more confident. When unsure, they, in keeping with the other subjects, opted for the equivocal category 3 (unsure, probably different) rather than category 4 (unsure, probably same). Subject 2 was especially conservative and, although clearly experiencing a change in perception for stimuli around $50 \%$ green, was unwilling to state categorically that the stimuli were of the same hue. 

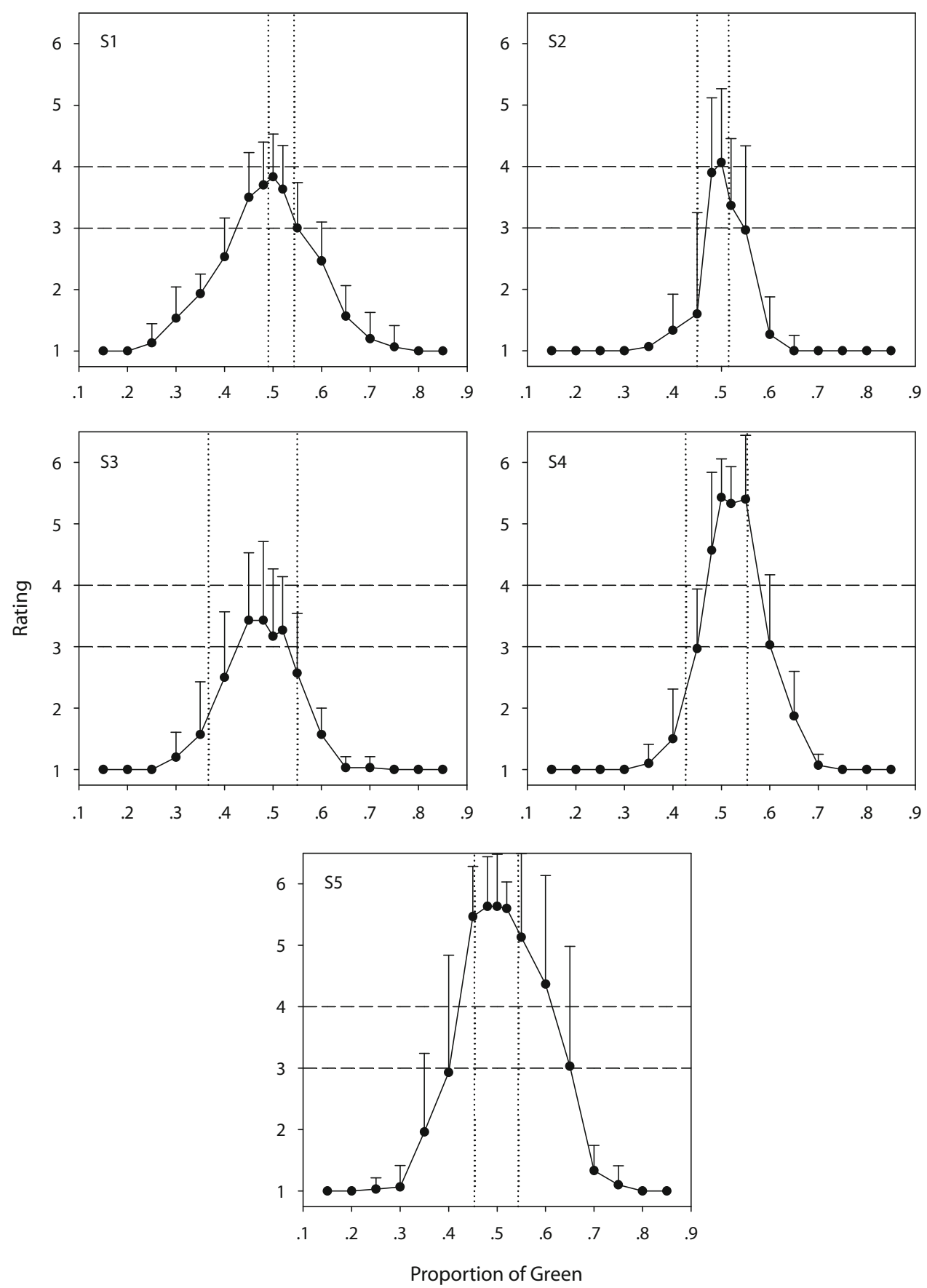

Figure 1. Average category ratings as a function of the proportion of green in the variable stimulus. Each panel represents an individual subject. The dotted vertical lines mark the edges of the matching range reported by the anomaloscope. The dashed horizontal lines delineate the equivocal rating categories unsure, probably different (rating 3) and unsure, probably same (rating 4). 
The automatic mode is the most likely choice for general color vision screening with the Roland IF-II anomaloscope. In this mode, the anomaloscope requires binary judgments. The effect of more or less conservative response tendencies, where ratings are not required, is uncertain, but it seems likely that individuals with a conservative response tendency will opt for a different response more frequently than will those with a liberal response tendency. Especially when only a few same responses are required to determine the borders of the MR, its width will reflect, to some extent, the response characteristics of the individual being tested, irrespective of the internal perceptual experience. Similar comments will apply to adjustment methods, since what is required for a match will be unique to the individual. Whatever method is used, response bias will be a factor; however, some methods will be less affected than others. An agreed-upon definition of MR is required, and adequate numbers of trials are necessary at the borders of the range. Large numbers of trials leave open the possibility of subject fatigue; therefore, an efficient adaptive psychophysical procedure is required to converge efficiently on the region of interest.

The fact that, in this study, despite displaying different degrees of conservatism, observers displayed a tendency to choose unsure, probably different over unsure, probably same is contradictory to previous research in other modalities (Macmillan \& Creelman, 2005; Stillman, Brown, $\&$ Troscianko, 2000). A possible reason for this may be that in the entire set of 510 trials, the probability of same pairs was relatively low. Response biases are known to vary with event probability (Green \& Swets, 1966/1974). If the reported MRs are presumed to be accurate, so that presentations within an individual's reported MR are considered to be events, the probability of same pairs would be in the range of .23-.35.

The outcomes of the study suggest that, notwithstanding the usefulness of anomaloscopic assessments for the diagnosis of abnormal color vision, there are some limitations with respect to investigations in which, for example, it is desirable to track small progressive changes in color vision over time. The psychophysical procedure currently employed by the Roland instrument, especially in its automatic mode, is likely to result in a reported MR and AQ that are confounded by the response biases of individuals, particularly over the few trials in the range of interest. Only two same judgments were required to define the border of the MR. It is likely that the numbers of trials required of observers using other instruments will not be adequate to minimize the effects of response bias. It should be possible for such instruments to employ more reliable adaptive psychophysical procedures to specify the borders of the MR with greater precision. The Color Vision Meter 712 anomaloscope employs a preferable psychophysical procedure determining a pair of psychometric functions. However, adaptive psychophysical procedures - for example, the staircase technique (Levitt, 1971) - are efficient in that they eliminate the need to obtain the entire psychometric function by targeting a specific point on it. In line with this approach, it would be useful to define the borders of the MR in terms of a chosen level of performance on either side of the expected 50/50 midpoint. The parameters of interest with the anomaloscope present particular measurement problems, because the intention is to establish which stimulus pairs are subjectively equivalent. This requirement is subtly different from determining which physical differences can be discriminated. In most adaptive psychophysical procedures, responses are scored correct if they accurately reflect an objectively quantifiable physical difference and incorrect if they do not. Changes in the stimulus can be arranged after a set number of correct or incorrect responses. In anomaloscopic testing, the observer's subjective experience of sameness or difference is, by definition, correct, and it is not possible to know how the individual's choice of response alternative corresponds to his or her perception. Therefore, the procedure needs to substitute the proportion of same (match) or different (not-a-match) responses for that of correct or incorrect responses in the adaptive procedure of choice. For a comparison among three candidate adaptive procedures, see Stillman (1989). An adaptive procedure establishing the percentage of green in the variable stimulus on either side of $50 \%$ green for, say, $79 \%$ same responses is one possible definition of the borders of the MR. An analogous definition could be adopted for Rayleigh red-green matches. It will also be desirable to decide upon a set of brightness values or to arrange a series of initial trials sampling a range of wavelength mixtures, to assign the brightness values for the testing to follow (see Sommerhalder, Pelizzone, \& Roth, 1997).

In reality, different instruments will not have equivalent optics and may be differentially affected by such variables as ambient, or internal, temperature fluctuations (Jordan \& Mollon, 1993). It seems unnecessary to add methodological variables to these impediments to intersubject and interstudy comparisons. If an operational definition of the MR could be agreed upon, it may be that only software changes would be required of manufacturers to ensure greater precision and improved validity of data obtained through anomaloscopic assessments. Standardized automatic adaptive testing, using appropriate psychophysical techniques, has the potential to improve the efficiency and accuracy of anomaloscopic assessments, beyond that obtained with the procedures currently employed.

\section{AUTHOR NOTE}

This research was supported by a grant from the New Zealand Dental Research Foundation. Correspondence concerning this article should be addressed to J. A. Stillman, School of Psychology, Massey University Albany, Private Bag 102-904, North Shore MSC, Auckland, New Zealand (e-mail: j.a.stillman@massey.ac.nz).

Note-Accepted by the editorial board of Editor-Elect Jeremy M. Wolfe.

\section{REFERENCES}

Green, D. M., \& Swets, J. A. (1974). Signal detection theory and psychophysics. Huntington, NY: Krieger. (Original work published 1966)

Jordan, G., \& Mollon, J. D. (1993). The Nagel anomaloscope and seasonal variation of colour vision. Nature, 363, 546-549.

LEVITT, H. (1971). Transformed up-down methods in psychoacoustics. Journal of the Acoustical Society of America, 49, 467-477.

Macmillan, N. A., \& Creelman, C. D. (2005). Detection theory: A user's guide (2nd ed.). Mahwah, NJ: Erlbaum. 
National ACADEmy of Sciences (1981). Procedures for testing color vision: Report of Working Group 41. Washington, DC: National Academies Press.

Nousiainen, I., Kälvï̈Inen, R., \& MäntyJäRvi, M. (2000). Color vision in epilepsy patients treated with vigabatrin or carbamazepine monotherapy. Ophthalmology, 107, 884-888.

Pelizzone, M., Sommerhalder, J., Roth, A., \& Hermès, D. (1993). Automated Rayleigh and Moreland matches: Optimization of stimulation parameters for normal observers. In B. Drum (Ed.), Colour vision deficiencies XI (Documenta Ophthalmological Proceeding Series, Vol. 56, pp. 345-355). Dordrecht: Kluwer.

Sommerhalder, J., Pelizzone, M., \& Roth, A. (1997). Stimulation parameters for automatic examination of color vision [Online]. Klin Monatsbi Augenheikd, 210, 319-324. [Abstract from: PubMed Indexed for MEDLINE. Item: PMID: 9324543]

Squire, T. J., Rodriguez-Carmona, M., Evans, A. D. B., \& BarBUR, J. L. (2005). Color vision tests for aviation: Comparison of the anomaloscope and three lantern types. Aviation, Space, \& Environmental Medicine, 76, 421-429.

Stillman, J. A. (1989). A comparison of three adaptive psychophysical procedures using inexperienced listeners. Perception \& Psychophysics, 46, 345-350.

Stillman, J. A. (2007). A psychophysical study of the validity of anomaloscopic assessments of colour vision in the blue-green range. In S. Mori \& W. Wong (Eds.), Fechner Day 2007: Proceedings of the 23rd Annual Meeting of the International Society for Psychophysics (pp. 465-470). Tokyo: International Society for Psychophysics.

Stillman, J. A., Brown, G. M., \& Troscianko, T. (2000). Influence of sensitivity on response bias in taste and audition. Perception \& Psychophysics, 62, 1645-1654.

(Manuscript received January 30, 2008; revision accepted for publication March 18, 2008.) 